\title{
Benefits and Limitations of TKIs in Patients with Medullary Thyroid Cancer: A Systematic Review and Meta-Analysis
}

\author{
Zoe A. Efstathiadou ${ }^{a}$ Charalambos Tsentidis ${ }^{b}$ Alexandra Bargiota ${ }^{c}$ \\ Vasiliki Daraki $^{d}$ Kalliopi Kotsa ${ }^{e}$ Georgia Ntali $^{f}$ Labrini Papanastasiou $^{g}$ \\ Stelios Tigas $^{h}$ Konstantinos Toulis $^{i} \quad$ Kalliopi Pazaitou-Panayiotou ${ }^{j}$ \\ Maria Alevizakik
}

a Department of Endocrinology, "Hippokration" General Hospital of Thessaloniki, Thessaloniki, Greece; ${ }^{b}$ Department of Endocrinology, General Hospital of Nikaia "Agios Panteleimon", Piraeus, Greece; 'Department of Endocrinology, University of Thessaly, Larisa, Greece; 'Department of Endocrinology, University Hospital of Crete, Heraklion, Greece; e Department of Endocrinology, "Ahepa" Hospital, Aristotle University, Thessaloniki, Greece; ${ }^{\text {DDepartment }}$ of Endocrinology, Diabetes and Metabolism, "Evangelismos" Hospital Athens, Athens, Greece; 9Department of Endocrinology and Diabetes Center, Athens General Hospital "G. Gennimatas", Athens, Greece; hepartment of Endocrinology, University of loannina, loannina, Greece; 'Department of Endocrinology, 424 Military Hospital, Thessaloniki, Greece; 'Division of Endocrinology, Endocrine Oncology, Interbalkan Medical Center, Thessaloniki, Greece; ${ }^{k}$ Endocrine Unit, Department of Medical Therapeutics, School of Medicine, Kapodistrian University of Athens, Athens, Greece

\section{Keywords}

Tyrosine kinase inhibitors · Medullary thyroid cancer ·

Tyrosine kinase

\begin{abstract}
Introduction: Tyrosine kinase inhibitors (TKIs) have been used in patients with advanced medullary thyroid carcinoma (MTC); however, data on their effectiveness and safety are limited. The aim of this systematic review and meta-analysis was to document clinical response and toxicities of TKIs in advanced MTC. Methods: We systematically searched major databases for articles or abstracts on TKI use in MTC patients until May 2018. Objective response (OR), defined as the sum of complete + partial response, expressed as percentage, was our primary endpoint, while disease stability, disease
\end{abstract}

progression (DP), median progression-free survival (PFS), and drug discontinuation rate due to adverse events (AEs) were secondary endpoints. Pooled percentages, PFS time, and $95 \%$ Cls were reported. Results: Thirty-three publications were finally included in the analysis: 1 phase IV, 2 phase III trials evaluating vandetanib and cabozantinib, respectively, 20 phase I or II studies, and the remaining 10 studies of retrospective-observational nature. OR was documented in $28.6 \%$ (95\% Cl 25.9-31.9) of patients. Stable disease was recorded in $46.2 \%(95 \% \mathrm{Cl} 43.3-49.1)$. Overall, DP was observed in $22.9 \%$ (95\% Cl 20.4-27.6). Grade 3 or more AEs occurred in $48.5 \%$ (95\% Cl 45.5-51.5) of patients, and drug discontinuation was reported in 44.7\% (95\% Cl 41.7-47.6). In general, use of TKIs conferred a PFS of 23.3 months $(95 \% \mathrm{Cl}$ 21.07-25.5). In particular, vandetanib induced an OR in $33.8 \%$ (95\% Cl 29.6-38.0) of patients and cabozantinib in 
27.7\% (95\% Cl 22.05-33.4). DP occurred in $23.7 \%$ (95\% Cl 19.9-27.6) with vandetanib use and in $22.6 \%(95 \% \mathrm{Cl} 17.4-$ 27.9) in cabozantinib-treated patients. Sorafenib, the third most frequently studied drug, showed intermediate efficacy, but higher discontinuation rates. Conclusion: Treatment with TKIs in MTC patients with progressive disease is associated with a moderate therapeutic benefit, with achievement of either disease stability or partial response in $73 \%$. The toxicity of these drugs is not negligible, but it is, nonetheless, manageable.

(c) 2020 European Thyroid Association Published by S. Karger AG, Basel

\section{Introduction}

Medullary thyroid cancer (MTC) is a malignant tumor arising from the thyroid parafollicular $\mathrm{C}$ cells. It is considered a rare neoplasm since it accounts for just $1-2 \%$ of all thyroid cancers $[1,2]$. Prognosis depends on disease extent at diagnosis. While curative surgery confers a 10year survival of $96 \%$ in localized disease, in the presence of advanced MTC, regional unresectable disease, or distant metastases, survival is significantly reduced $[2,3]$. In patients with distant metastases, median overall survival does not exceed 3 years [3]. Consequently, for those cases not cured by surgery, there is a need for further effective treatment.

The disease is sporadic in $70-80 \%$ of cases and hereditary in $20-30 \%$ [2, 4]. RET (rearranged during transfection) proto-oncogene mutations play a key role in the pathogenesis of the disease. RET encodes a single-pass transmembrane protein receptor that belongs to receptor tyrosine kinase family. Activating germline mutations are present in almost all cases of hereditary MTC, whereas somatic mutations of RET are found in about $50 \%$ of sporadic cases $[1,5,6]$.

The understanding of molecular mechanisms that are involved in the pathogenesis of MTC resulted in remarkable progress in the development of therapeutic strategies [7]. Specific mutations are linked to more aggressive disease, as is the case with codon M918T mutation that affects the tyrosine kinase domain of the receptor and causes constitutive receptor activation. When the M918T mutation is in the germline in the context of MEN2B syndrome, it is linked to very early MTC occurrence and aggressive, metastatic behavior. Similar behavior is observed in sporadic MTC with somatic M918T mutation.

The intracellular signaling pathways triggered by RET activation include the RAS-RAF-MEK-ERK and the PI3K-AKT-mTOR. Canonical VEGFR signaling follows the same intracellular signaling pathways and plays a central role in the effectiveness of targeted therapies, as well as in the associated side effects $[3,7]$.

Tyrosine kinase inhibitors (TKIs) are small molecules that target intracellular signaling pathways at different sites and, to date, they are the mainstay treatment for advanced MTC. Cabozantinib and vandetanib inhibit multiple kinases including RET and VEGFR. Among others, signaling pathways initiated by both RET and VEGFR are important for MTC development and progression [3, 7]. Although promising, these drugs show only a modest effect on advanced thyroid cancer, while they exhibit significant toxicity [8]. A recent meta-analysis has shown a clinical benefit associated with vandetanib and to a lesser degree with cabozantinib [8]. Since that publication, numerous further studies have been published including the results from a phase III trial [9].

The aim of the present study was to further clarify the role of TKIs in advanced MTC by estimating the effectiveness expressed by different indices of tumor response and by addressing the limitations of TKI use related to the toxicity of the different TKIs studied so far. To this end, we performed a meta-analysis of all available studies reporting data on efficacy and safety of TKI use in advanced MTC.

\section{Materials and Methods}

\section{Search Strategy}

The present study was conducted according to Preferred Reporting Items for Systematic Reviews and Meta-Analyses (PRISMA) guidelines. The research question was dual. First, whether and to what degree, TKI use in advanced MTC results in tumor response by RECIST (Response Evaluation Criteria in Solid Tumors) criteria [10]. Second, what is the drug discontinuation rate due to treatment-related toxicities. Both questions were examined for TKIs as a drug class and then, specifically for each compound tested so far. The review protocol was registered in the international prospective register of systematic reviews (PROSPERO) with reg. No. CRD42018083924.

A computerized literature search in MEDLINE, EMBASE, and Scopus covering the period until May 2018 was performed. The citation lists of all relevant publications and review articles were hand-searched. No language limitations were applied.

The keywords used for the disease "(medullary thyroid cancer OR medullary thyroid carcinoma)" were entered as free-text terms, in an attempt to maximize the sensitivity of the search strategy and indeed they rendered the largest amount of results.

For the intervention part, generic names of TKIs known to have been used for MTC along with the general term in various combinations were used. In addition, a wide search for relative compounds ever tried on MTC was performed in "ClinicalTrials.gov" database, and another 6 compounds emerged, one of which with 3 different names. In detail, the intervention part was formed as fol- 
Fig. 1. Flow diagram of study selection.

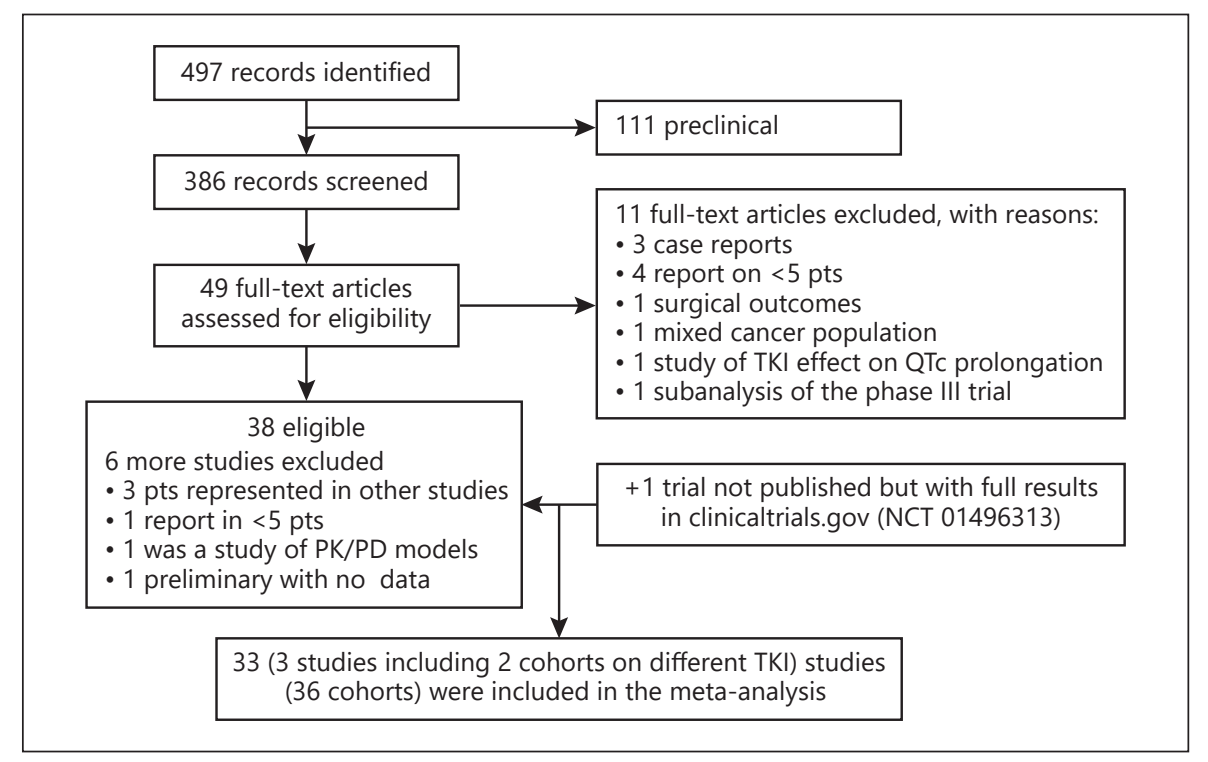

lows: ("tyrosine kinase inhibitors" OR" tyrosine kinase inhibitor" OR tki OR tkis OR "Multikinase inhibitors" OR "Multikinase inhibitor" OR "Protein Kinase Inhibitors" OR afatinib OR alectinib OR axitinib OR bosutinib OR cabozantinib OR crizotinib OR dasatinib OR erlotinib OR imatinib OR ibrutinib OR lapatinib OR midostaurin OR neratinib OR nilotinib OR pacritinib OR pazopanib OR ponatinib OR regorafenib OR sorafenib OR sunitinib OR vandetanib OR ziv-aflibercept OR gefitinib OR motesanib OR XL184 OR avastin OR bevacizumab OR alvocidib OR orantinib OR selumetinib OR semaxinib OR vatalanib OR AP24534 OR anlotinib OR nintedanib OR larotrectinib OR bortezomib OR sulfatinib OR tipifarnib OR LOXO-101 OR LOXO-292).

\section{Selection of Studies}

Studies were included in the meta-analysis if they presented extractable results on tumor response to TKIs, or on adverse events (AEs) of TKI therapy in MTC. Reports of individual cases or series of $<5$ patients were excluded.

\section{Data Extraction}

Data extraction was performed independently by 4 groups of 2 reviewers each. The following data were recorded from each of the eligible studies: demographic data (citation data, country, study period, study drug, number of patients, age, sex, and duration of treatment), drug use data (initial dose, dose reduction or interruption, drug discontinuation, and reason for discontinuation), drug efficacy (complete, partial, objective response, stable disease, and disease progression), and finally, data on drug toxicity (AEs of any grade and grade $\geq 3$ ).

Parameters of tumor response that were extracted from each study according to the revised RECIST system [10] were the following: complete response (CR) - complete regression of lesions, partial response $(\mathrm{PR})$ - a decrease of at least $30 \%$ of the sum of the longest diameter of target lesions, stable disease (SD) - any response between a $30 \%$ decrease and a $20 \%$ increase in size of target lesions, progressive disease (PD) - an increase of at least $20 \%$, and objective response $(\mathrm{OR}=\mathrm{PR}+\mathrm{CR})$.
Any disagreement between reviewers responsible for data extraction was resolved by discussion. For studies with missing data or data presented in conjunction with other types of differentiated thyroid cancer, we proceeded to communication with the authors requiring online supplementary data when available.

\section{Outcomes}

The primary endpoint was OR rate; disease stability, median progression-free survival (PFS), drug discontinuation due to AEs, and grade $\geq 3$ AEs (G3AEs) were secondary endpoints. Patients with drug interruption or dose reduction due to AEs were not included in the drug discontinuation outcome. Recorded AEs included cases with hand-foot syndrome, diarrhea, nausea/vomiting, arterial hypertension, fatigue/asthenia, abnormal liver tests, mucositis, rash, anorexia, abdominal pain, constipation, weight loss, hematological disorders, cardiac disorders, renal disorders, cough/dyspnea, dysphonia, headache, peripheral edema, arthralgia/myalgia, musculoskeletal pain, and increased thyroid stimulating hormone levels.

\section{Quantitative Data Synthesis}

Pooled percentages were calculated using meta-analysis of binomial data. Direct comparisons between specific drugs were not feasible and drug-specific effect estimates are provided descriptively. Data are presented as percentages with 95\% CIs. PFS periods are presented as months with 95\% CIs in parentheses.

Heterogeneity across studies was assessed using Cochran's Q $\chi^{2}$ test and $I^{2}$ statistic. A $p$ value $<0.05$ or $I^{2}>50 \%$ was considered as significant heterogeneity. Since substantial heterogeneity was observed, we used random-effects models to summarize pooled data. The DerSimonian and Laird method was used for the estimation of summarized effect size in each subanalysis.

STATA for Windows version 15 (Stata Corp, TX, USA, 2017) was used for statistical processing. 
Table 1. Study characteristics by drug received

\begin{tabular}{|c|c|c|c|}
\hline Drug & Cohorts, $n$ & Type of study & Patients \\
\hline Axitinib & 3 & 1 retrospective, 2 phase II & 17 \\
\hline Dovitinib & 1 & 1 phase II & 12 \\
\hline Imatinib & 3 & 1 phase II, 2 open label & 30 \\
\hline Motesanib & 1 & 1 phase II in 3 papers & 91 \\
\hline Pazopanib & 1 & 1 phase II & 35 \\
\hline Sorafenib & 8 & 2 phase II, 4 retrospective, 1 observational, 1 off label & 99 \\
\hline Sunitinib & 2 & 2 phase II & 33 \\
\hline Sorafenib OR sunitinib & 1 & 1 retrospective & 7 \\
\hline \multirow[t]{2}{*}{ Sorafenib + tipifarnib } & 1 & 1 phase I & 13 \\
\hline & 36 cohorts in 33 studies & $\begin{array}{l}2 \text { phase III, } 12 \text { phase II, } 1 \text { phase I-II, } 2 \text { phase } \\
\text { I, } 3 \text { open label, } 1 \text { off label, } 9 \text { retrospective, } 1 \text { observational }\end{array}$ & 1,274 \\
\hline
\end{tabular}

\section{Results}

\section{Characteristics of Eligible Studies}

A total of 497 studies were identified using the aforementioned search strategy. Refining the search in studies conducted in humans returned 386 clinical studies (Fig. 1).

Thirty-three publications met the inclusion criteria for analysis [9, 11-42]: 1 phase IV, 2 phase III trials evaluating vandetanib and cabozantinib respectively, 20 phase I or II studies, and the remaining 10 studies of retrospective-observational nature. Vandetanib is represented with 12 studies including 498 individuals, whereas cabozantinib with 2 studies including 367 individuals, most likely because the former was the first to be approved for use in MTC. In total, data of 1,274 patients were evaluated, 81 in phase IV trials, 561 in phase III trials, 441 in phase I-II trials, and 191 in retrospective studies (Table 1; online suppl. Table 1; for all online suppl. material, see www.karger.com/doi/10.1159/000509457). Significant heterogeneity was detected in all effect estimates. Among the 36 cohorts studied in the 33 studies, 20 cohorts included patients with locally advanced or metastatic disease, 13 included patients with PD and, in 3 cohorts described in 2 studies, patients were recruited for PD and/ or symptoms. Data on the proportion of sporadic versus familial MTC were not reported in all studies (online suppl. Table 1). In total, 164 cases were documented as familial MTC.

\section{Quality Assessment of Eligible Studies}

The methodological quality assessment is presented in online supplementary Table 1.

\section{Overall Effects}

Overall, 29 studies provided data on objective response $(\mathrm{OR}=\mathrm{CR}$ and PR). OR was documented in $28.6 \%$ (95\% CI 25.9-31.9; Cochran's $\mathrm{Q}=259.9, \chi^{2}$ [df 28], $p<0.001$, $I^{2}=89.2 \%$ ) of the patients (test for overall effect $z=21.05$, $p<0.001$ ). Regarding drugs with phase III and IV studies, OR was observed in $27.7 \%$ (95\% CI 22.05-33.4) with cabozantinib and in $33.8 \%$ (95\% CI 29.6-38.0) with vandetanib use (Fig. 2). Apart from these 2 molecules, sorafenib appeared to be the next more extensively tested, in 8 trials ( 2 phase II, 4 retrospective, one observational and one off label), on a total of 99 patients, with an OR of $27.5 \%$ (95\% CI 17.8-37.2). Control group data were avail-

Fig. 2. Data on objective response (OR) were provided by 29 studies. OR with TKIs in MTC was documented in 28.6\% (95\% CI 25.9-31.9; Cochran's Q $=259.9, \chi^{2}$ [df 28], $p<0.001, I^{2}=89.2 \%$; test for overall effect $z=21.05, p<0.001$ ).

(For figure see next page.) 
axitinib

Capdevila, 2017

Cohen, 2008

Subtotal $\left(I^{2}=0.0 \%, p=0.782\right)$

cabozantinib

Elisei, 2012

Kurzrock, 2011

Subtotal $\left(I^{2}=0.0 \%, p=0.921\right)$

dovitinib

Lim, 2015

Subtotal $\left(I^{2}=. \%, p=.\right)$

lenvatinib

Schlumberger, 2015

Subtotal $\left(R^{2}=. \%, p=\right.$.)

motesanib

Schlumberger, 2009

Subtotal $\left(R^{2}=. \%, p=\right.$.)

pazopanib

Bible, 2014

Subtotal $\left(R^{2}=. \%, p=.\right)$

sorafenib

Ito, 2017

Benekli, 2015

Frank-Raue, 2010

Lam, 2010

Capdevila, 2012

Ahmed, 2011

Subtotal $\left(R^{2}=52.4 \%, p=0.062\right)$

sorafenib, sunitinib

Massicotte (subgroup1), 2014

Subtotal $\left(R^{2}=. \%, p=\right.$.)

sorefenib+tipifarnib

Hong , 2011

Subtotal $\left(I^{2}=. \%, p=\right.$.)

sunitinib

Ravaud, 2017

Carr L, 2010

Subtotal $\left(R^{2}=64.0 \%, p=0.096\right)$

vandetanib

Werner, 2015

Tiedje, 2016

Uchino, 2017

Chougnet, 2015

Massicotte (subgroup2), 2014

NCT01496313 (subgroup 1), 2017

NCT01496313 (subgroup 2), 2017

Kraft, 2018

Robinson, 2010

Wells, 2010

Wells, 2012

Subtotal $\left(R^{2}=74.3 \%, p=0.000\right)$

Overall $\left(K^{2}=89.2 \%, p=0.000\right)$
$23.08(5.03,53.81)$

$18.18(2.28,51.77)$

1.18

1.00

2.17

$27.85(22.02,34.29) \quad 19.84$

$27.03(13.79,44.11) \quad 3.35$

$27.73(22.05,33.42) \quad 23.19$

$16.67(2.08,48.41) \quad 1.09$

$16.67(-6.50,39.83) \quad 1.09$

$35.59(23.55,49.13) \quad 5.34$

$35.59(22.80,48.38) \quad 5.34$

$2.20(0.26,7.71)$

$2.20(-1.53,5.92)$

8.24

8.24

$14.29(4.80,30.25) \quad 3.17$

$14.29(1.56,27.01) \quad 3.17$

$25.00(3.18,65.08) \quad 0.72$

$25.00(7.26,52.37) \quad 1.45$

$60.00(14.66,94.72) \quad 0.45$

$9.52(1.17,30.37) \quad 1.90$

$46.67(21.26,73.41) \quad 1.36$

$26.67(7.78,55.10) \quad 1.36$

$27.50(17.78,37.22) \quad 7.25$

$14.29(0.36,57.87) \quad 0.63$

$14.29(-14.47,43.04) \quad 0.63$

$38.46(13.85,68.42) \quad 1.18$

$38.46(11.18,65.75) \quad 1.18$

$38.46(20.22,59.42) \quad 2.36$

$71.43(29.04,96.33) \quad 0.63$

$45.45(28.44,62.47) \quad 2.99$

$9.52(1.17,30.37) \quad 1.90$

$50.00(18.70,81.29) \quad 0.91$

$35.71(12.75,64.86) \quad 1.27$

$21.67(12.07,34.19) \quad 5.43$

$36.36(10.92,69.20) \quad 1.00$

$20.00(9.05,35.64) \quad 3.62$

$29.27(16.12,45.53) \quad 3.71$

$29.41(10.31,55.95) \quad 1.54$

$15.79(3.38,39.57) \quad 1.72$

$20.00(7.71,38.56) \quad 2.72$

$45.02(38.49,51.68) \quad 20.92$

$33.81(29.60,38.01) \quad 44.75$

$28.62(25.96,31.29) \quad 100.00$ 
axitinib

Capdevila, 2017

Locati, 2014

Cohen, 2008

Subtotal $(I 2=73.3 \%, p=0.024)$

cabozantinib

Elisei, 2012

Kurzrock, 2011

Subtotal $\left({ }^{2}=0.0 \%, p=0.575\right)$

dovitinib

Lim, 2015

Subtotal $\left(I^{2}=. \%, p=.\right)$

imatinib

Frank-Raue, 2007

Gross, 2006

de Groot, 2007

Subtotal $\left(R^{2}=0.0 \%, p=0.509\right)$

lenvatinib

Schlumberger, 2015

Subtotal $\left(R^{2}=. \%, p=\right.$.)

motesanib

Schlumberger, 2009

Subtotal $\left(R^{2}=. \%, p=\right)$

pazopanib

Bible, 2014

Subtotal $\left(R^{2}=. \%, p=\right)$

sorafenib

Castroneves, 2016

Ito, 2017

Benekli, 2015

Frank-Raue, 2010

Lam, 2010

Capdevila, 2012

Ahmed, 2011

Subtotal $\left(R^{2}=51.2 \%, p=0.056\right)$

sorafenib, sunitinib

Massicotte (subgroup1), 2014

Subtotal $\left(R^{2}=. \%, p=\right.$.)

sorefenib+tipifarnib

Hong , 2011

Subtotal $\left(R^{2}=. \%, p=\right.$.)

sunitinib

Ravaud, 2017

Subtotal $\left(R^{2}=. \%, p=\right.$.)

vandetanib

Werner, 2015

Tiedje, 2016

Uchino, 2017

Chrisoulidou( subgroup 2), 2015

Chougnet, 2015

Massicotte (subgroup2), 2014

NCT01496313 (subgroup 1), 2017

NCT01496313 (subgroup 2), 2017

Kraft, 2018

Robinson, 2010

Wells, 2010

Wells, 2012

Subtotal $\left(I^{2}=58.6 \%, p=0.005\right)$

Overall $\left(R^{2}=48.5 \%, p=0.001\right)$

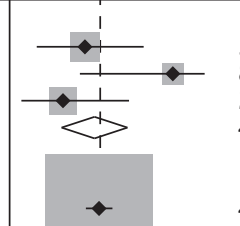

$38.46(13.85,68.42)$

$\begin{array}{ll}1.53 \\ 27.27(6.02,60.97) & 0.52\end{array}$

$43.33(26.54,60.12) \quad 2.61$

$45.66(38.93,52.50) \quad 19.04$

$40.54(24.75,57.90) \quad 3.22$

$44.92(38.64,51.20) \quad 22.26$

$58.33(27.66,84.83) \quad 1.04$

$58.33(29.75,86.92) \quad 1.04$

$11.11(0.03,48.24) \quad 0.78$

$33.33(4.32,77.72) \quad 0.52$

$26.67(7.78,55.10) \quad 1.30$

$23.33(7.65,39.02) \quad 2.61$

$44.07(31.15,57.59) \quad 5.13$

$44.07(30.85,57.29) \quad 5.13$

$48.35(37.74,59.07) \quad 7.91$

$48.35(37.69,59.02) \quad 7.91$

$57.14(39.35,73.67) \quad 3.04$

$57.14(39.98,74.30) \quad 3.04$

$76.92(46.18,94.96)$

$50.00(15.70,84.29) \quad 0.70$

$25.00(7.26,52.37) \quad 1.39$

$40.00(5.27,85.33) \quad 0.43$

$42.86(21.81,65.97) \quad 1.83$

$40.00(16.33,67.71) \quad 1.30$

$66.67(38.38,88.17)$

$48.39(38.48,58.29) \quad 8.09$

$71.43(29.04,96.33) \quad 0.61$

$71.43(37.78,105.07) \quad 0.61$

$30.77(9.09,61.42) \quad 1.13$

$30.77(4.60,56.93)$

$50.00(29.92,70.07)$

$50.00(29.93,70.07) \quad 2.26$

$61.90(38.43,81.89) \quad 1.83$

$30.00(6.67,65.24) \quad 0.87$

$57.14(28.86,82.33) \quad 1.22$

$25.00(0.06,80.58) \quad 0.35$

$55.00(41.61,67.87) \quad 5.22$

$54.55(23.37,83.25) \quad 0.96$

$52.50(36.12,68.48) \quad 3.48$

$56.10(39.74,71.53) \quad 3.57$

$11.76(1.45,36.44) \quad 1.48$

$52.63(28.86,75.55) \quad 1.65$

$53.33(34.32,71.65) \quad 2.61$

$41.99(35.54,48.64) \quad 20.09$

$46.79(42.33,51.24) \quad 43.30$

$46.26(43.34,49.18) \quad 100.00$

3

(For legend see next page.) 
able only by the 2 RCTs of vandetanib and cabozantinib. OR was documented in $13 \%$ (95\% CI 5.95-20.05) of control group patients (test for overall effect $z=3.61, p<$ 0.001; online suppl. Fig. 1).

Data on SD were provided by 34 studies. SD was reported in 46.2\% (95\% CI 43.3-49.1; $\mathrm{Q}=64.8, \chi^{2}(\mathrm{df} 33)$, $\left.p<0.001, I^{2}=48.5 \%\right)$ of patients $(z=31.02, p<0.001)$. In patients treated with cabozantinib, SD was reported in 44.9\% (95\% CI 38.6-51.2), while for vandetanib SD was reported in $46.7 \%$ of patients (95\% CI 42.3-51.2; Fig. 3). As for sorafenib, SD was achieved in $48.4 \%$ (95\% CI $38.5-$ 58.3 ; Fig. 3). SD was also reported in $52.1 \%$ of control patients (95\% CI 45.03-59.2; Q $=52.1, \chi^{2}$ [df 1$], I^{2}=57.6 \%$, $p=0.124$; test for overall effect $z=2.36, p<0.001$; online suppl. Fig. 2).

Twenty-seven studies reported data on overall disease progression (DP), which was documented in $22.9 \%$ (95\% CI 20.4-27.6; $\mathrm{Q}=173.7, \chi^{2}$ (df 26), $p<0.001, I^{2}=85 \%$ ) of patients $(z=17.8, p<0.001)$. Overall DP was recorded in $22.67 \%$ (95\% CI 17.4-27.9) of patients who received cabozantinib and in $23.78 \%$ (95\% CI 19.9-27.6) of vandetanib-treated patients (Fig. 4). DP was recorded in 19.2\% (95\% CI 10.4-28.0) of sorafenib-treated patients. In contrast, DP in MTC control patients was documented in 47.87\% (95\% CI 40.9-54.8; Q = 0.7, $\chi^{2}\left[(\mathrm{df} 1], I^{2}=0 \%\right.$, $p=0.4 ; z=13.4, p<0.001$; online suppl. Fig. 3$)$.

Data regarding drug discontinuation due to toxicity or DP were found in 26 studies. Overall, 44.7\% (95\% CI 41.7-47.6; Q = 315.1, $\chi^{2}$ [df 25], $\left.I^{2}=92.1 \%\right)$ of patients discontinued the drug $(z=29.6, p<0.001)$. In the cabozantinib group, that proportion was $54.8 \%$ (95\% CI $47.9-$ $61.6)$, while in the vandetanib group, it was $39.7 \%$ (95\% CI 35.3-44.1) and in the sorafenib group, 32.3 (95\% CI 24.3-40.4; Fig. 5). Even more, $78.6 \%$ of MTC control patients discontinued the drug $(95 \% \mathrm{CI} 72.9-84.3 ; \mathrm{Q}=6.43$, $\chi^{2}(\mathrm{df} 1), I^{2}=84.4 \%, p=0.011 ; z=26.9, p<0.001$; online suppl. Fig. 4).

Usable data on G3AEs were collected from 26 studies. G3AEs were reported in 48.5\% (95\% CI 45.5-51.5; Q = 139.7, $\chi^{2}$ (df 25), $\left.p<0.001, I^{2}=82.1 \%\right)$ of patients $(z=$ $31.9, p<0.001)$. That proportion was $66.7 \%$ (95\% CI 60.7-72.7) for cabozantinib, while in the vandetanib group it was 39.6\% (95\% CI 35.0-44.2; Fig. 6). Interestingly, G3 AEs were reported in $23.7 \%$ of MTC control patients (95\% CI 17.8-29.5; $\mathrm{Q}=10.4, \chi^{2}(\mathrm{df} 1), p=0.001$,

Fig. 3. Stable disease (SD) data were provided by 34 studies. SD with TKIs in MTC was reported in $46.2 \%$ of patients (95\% CI $43.3-$ 49.1; $\mathrm{Q}=64.08, \chi^{2}$ [df 33], $p<0.001, I^{2}=48.5 \%$; test for overall effect $z=31.02, p<0.001$ ).

TKIs in Medullary Thyroid Cancer
$I^{2}=90.4 \% ; z=7.9, p<0.001 ;$ online suppl. Fig. 5$)$. There is no obvious explanation for this finding. One speculation is that the adverse effects are due to the tumor itself or alternatively to other medications probably administered to these patients (not reported).

PFS data were evident in 16 studies. Overall, PFS was 23.3 months (95\% CI 21.7-25.5; $\mathrm{Q}=816.1, \chi^{2}$ [df 15], $p<$ $\left.0.001, I^{2}=98.2 \% ; z=20.5, p<0.001\right)$. PFS in vandetanibtreated patients was 33.3 months (95\% CI 28.4-38.1), while for the cabozantinib group, it was 11.2 months (95\% CI 8.3-14.5; Fig. 7). For sorafenib, PFS was 12.4 months (95\% CI 8.4-16.4), while PFS in MTC control patients was 11.2 months $\left(95 \%\right.$ CI 9.2-13.2; $\mathrm{Q}=60.3, \chi^{2}[\mathrm{df}$ $1], p<0.001, I^{2}=98.3 \% ; z=11.05, p<0.001$; online suppl. Fig. 6).

\section{Sensitivity Analysis}

Furthermore, we performed sensitivity analyses excluding those studies with $<10$ patients in order to evaluate whether the observed findings would be different from the initial analysis. Interestingly, there were minimal changes in each overall effect size, which remained practically unchanged (online suppl. Fig. 7-12).

Separate analyses in the 2 subpopulations of sporadic and familial MTC were not feasible, because of the paucity of information reported in most studies and the subsequent lack of statistical power.

\section{Discussion}

In this meta-analysis, we explored the effects of all TKIs that have been used in the treatment of advanced MTC. In general, evidence of OR was observed in about one-fourth $(28.6 \%)$ of the patients. Of note, CR has not been documented in any patient with advanced MTC. Disease stability was achieved in $46.2 \%$ of treated patients. The 2 TKIs that are currently licensed for advanced MTC, vandetanib and cabozantinib, were both found to have notable PR rates of 33.8 and $27.7 \%$, respectively, even though the latter was studied in patients at a more advanced disease stage.

However, TKIs are associated with considerable toxicity with AEs of grade 3 or higher occurring in approximately half of the treated patients, leading to drug discontinuation. The incidence of G3AEs in vandetanib and cabozantinib-treated patients was 39.6 and $66.7 \%$, respectively. Accordingly, drug discontinuation due to either toxicity or DP was recorded in $39.7 \%$ of vandetaniband in $54.8 \%$ of cabozantinib-treated subjects. 


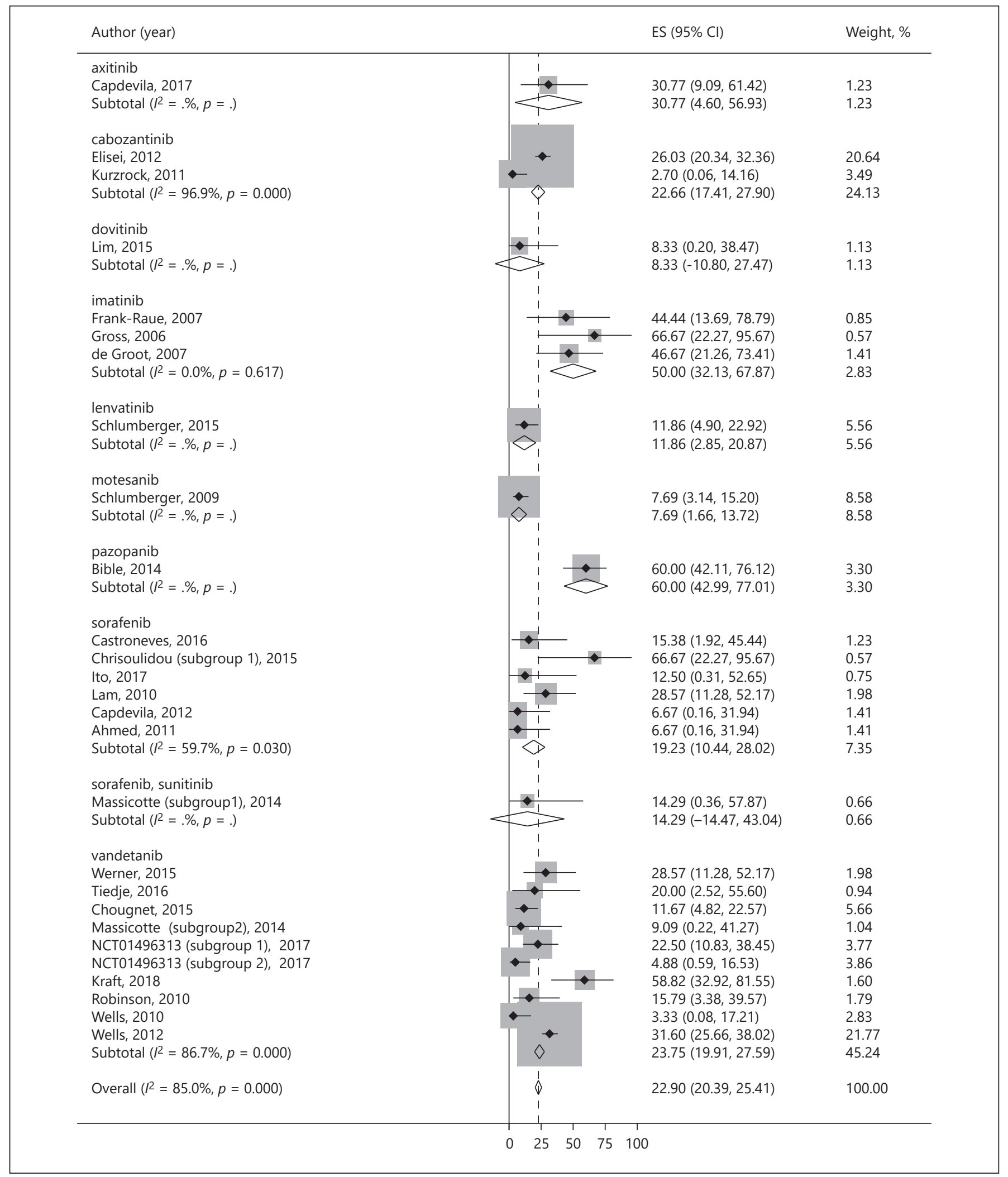

Fig. 4. Twenty-seven studies reported data on overall disease progression (DP). DP with TKIs in MTC was documented in $22.9 \%$ of patients (95\% CI 20.4-27.6; $\mathrm{Q}=173.7, \chi^{2}$ [df 26], $\left.p<0.001, I^{2}=85 \% ; z=17.8, p<0.001\right)$. 


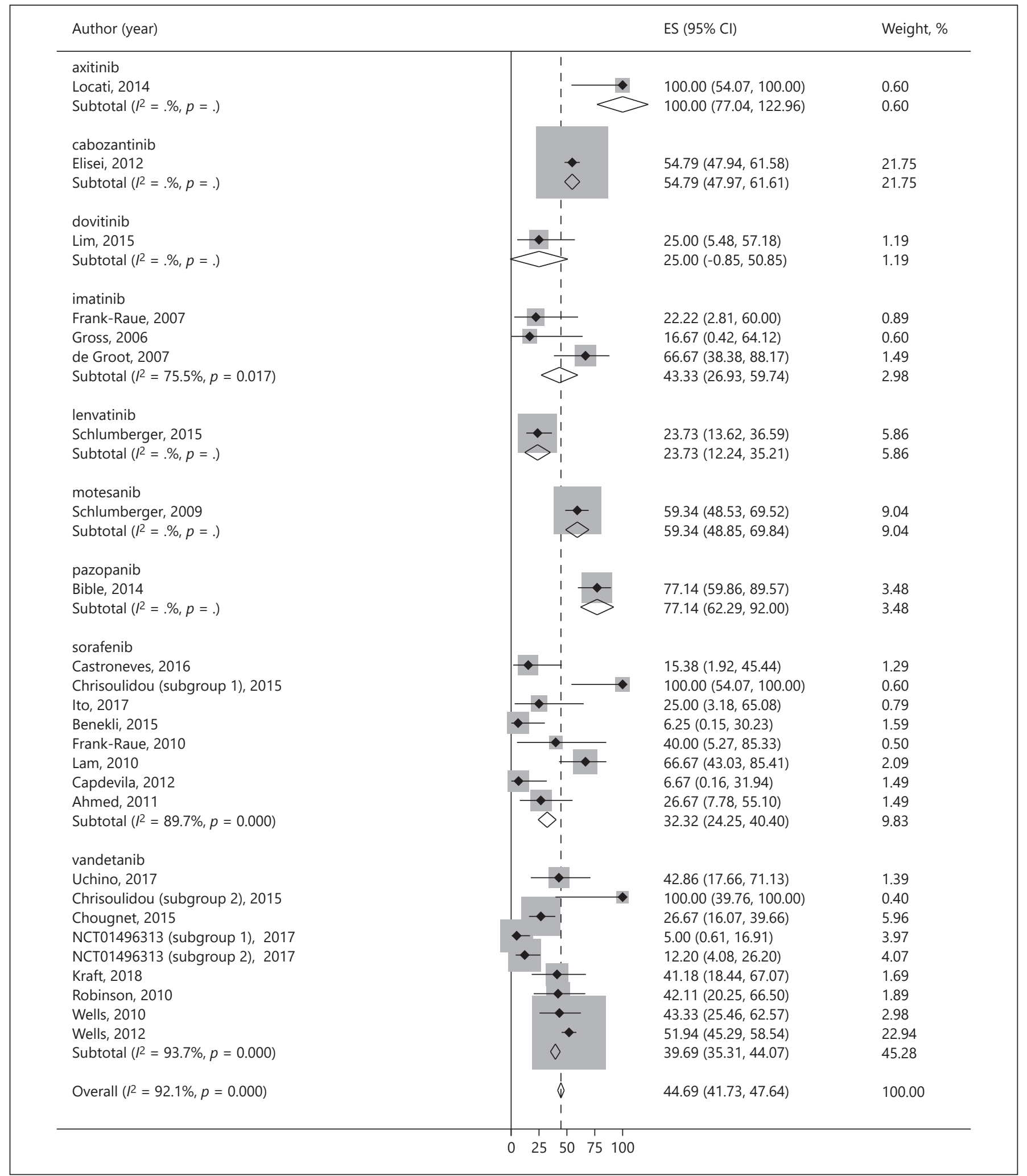

Fig. 5. Data regarding drug discontinuation due to toxicity or disease progression were found in 26 studies that reported therapy with TKIs in MTC patients. Overall, 44.7\% (95\% CI 41.7-47.6; Q = 315.1, $\chi^{2}$ [df 25], $p<0.001$, $\left.I^{2}=92.1 \%\right)$ of patients discontinued the drug $(z=29.6, p<0.001)$. 


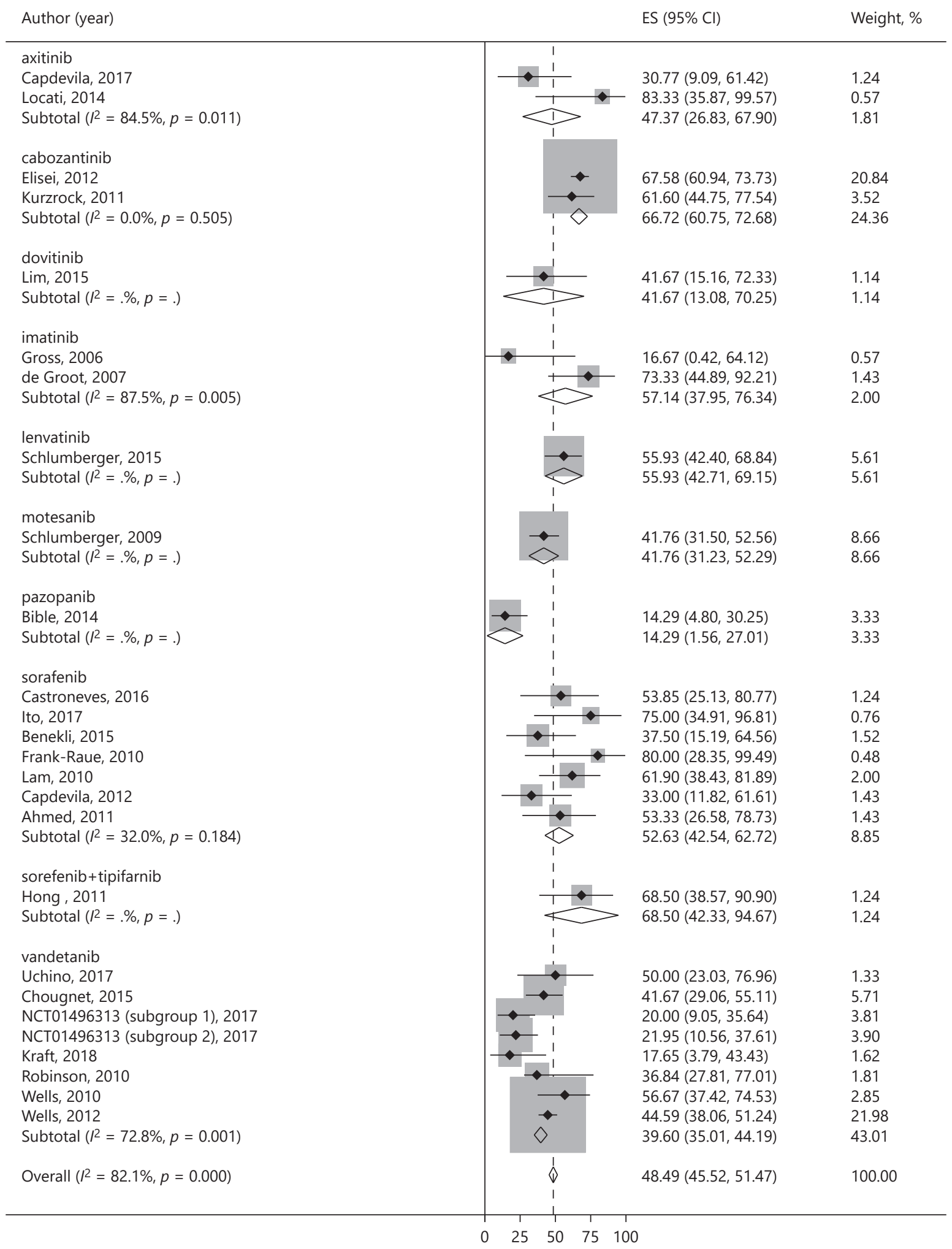

Fig. 6. Usable data on grade $\geq 3$ adverse events (G3AEs) were collected from 26 studies. G3AEs were reported in 48.5\% (95\% CI 45.5-51.5; $\left.\mathrm{Q}=139.7, \chi^{2}[\mathrm{df} 25], p<0.001, I^{2}=82.1 \%\right)$ of patients $(z=31.9, p<0.001)$. 


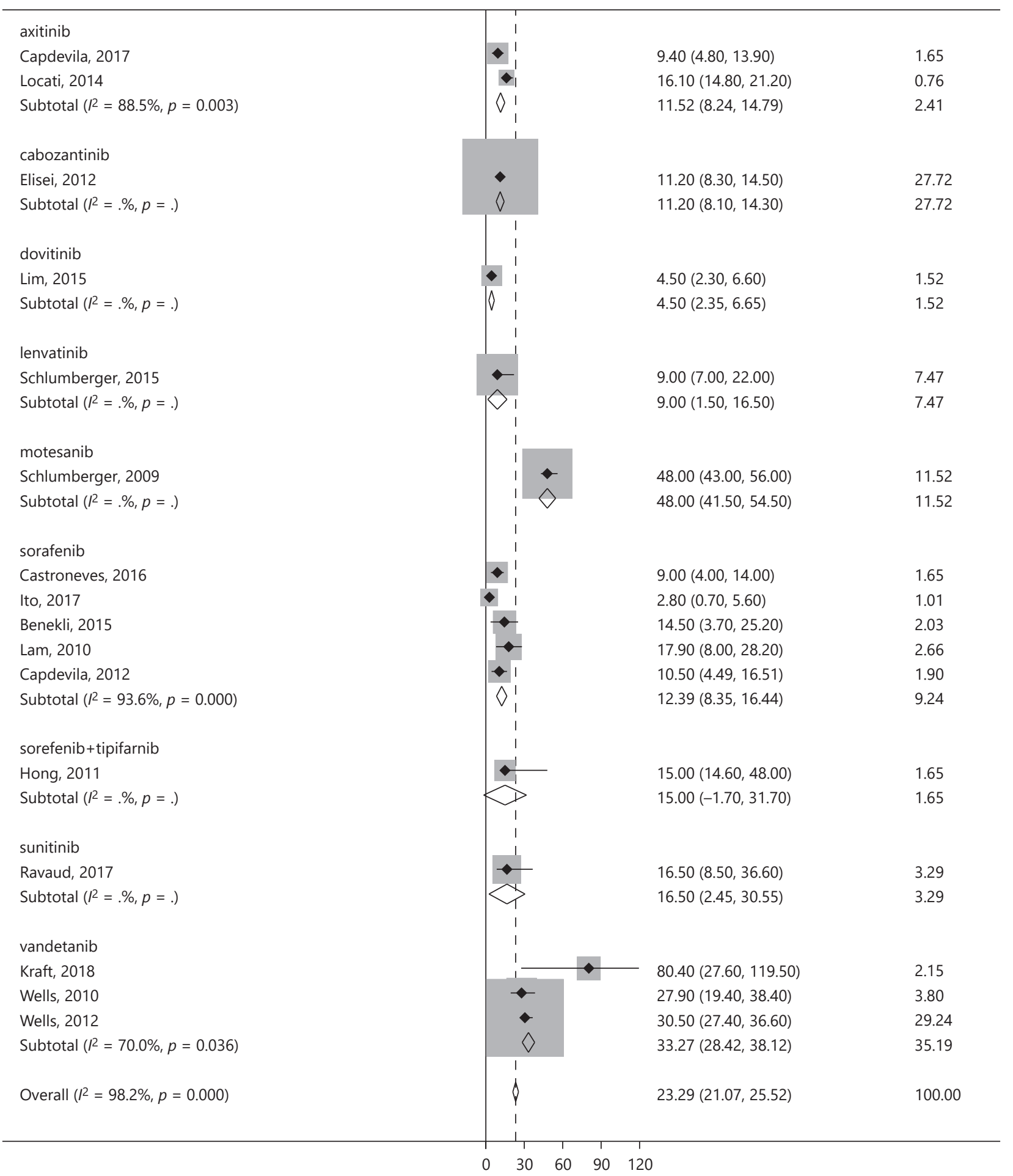

Fig. 7. Progression-free survival (PFS) data were evident in 16 studies. Overall, PFS was 23.3 months (95\% CI 21.07-25.5; $\left.\mathrm{Q}=816.1, \chi^{2}[\mathrm{df} 15], p<0.001, I^{2}=98.2 \% ; z=20.5, p<0.001\right)$. 
To the best of our knowledge, there are only 2 metaanalyses that have reviewed the effects of TKIs in MTC [8, 43].

The first included 16 studies of different TKI molecules [8]. In that meta-analysis, OR was estimated somewhat higher for vandetanib-treated (40\%) and similar for cabozantinib-treated (27\%) patients. It should be noted that the inclusion criteria had been more strict in that analysis compared to ours, as it comprised only 3 studies (280 patients) with vandetanib, 2 studies (256 patients) with cabozantinib, whilst a recent phase III study [9] of cabozantinib was not included in the analyses.

The second examined the efficacy of vandetanib on locally advanced MTC in 10 studies, including a study in pediatric patients with multiple endocrine neoplasia $2 \mathrm{~B}$, where the existence of specific genetic mutations might represent a considerable modifier of the pharmacological effect [43]. Furthermore, in the present meta-analysis, we have included 3 more patient cohorts with an additional input of 32 patients on vandetanib [29, 32, 42].

In more recent years, new publications have emerged, reporting on "real life" use of these agents in progressive MTC, that is, outside clinical trials, with longer follow-up, which thus might shed more light in the clinical use of these agents. Therefore, the present meta-analysis, following a more inclusive approach and thus resulting in the addition of a much larger number of patients and a broader set of TKIs might provide a more representative estimate of the benefits and the adverse effects of TKIs in MTC.

Data might suggest a better response rate and fewer G3AEs with vandetanib than cabozantinib. However, this observation should be interpreted with caution considering the differences in study design of the phase III studies $[9,26]$. In the cabozantinib trial, subjects were included in the study after documented DP by RECIST criteria, whereas in the vandetanib trial, subjects had a more indolent disease as indicated by better survival rates in the control group compared to cabozantinib study (19.3 vs. 4 months). Of note, despite the previous observation, $\mathrm{PD}$ rates were comparable or slightly higher in vandetanibtreated $(23.7 \%)$ than in cabozantinib-treated $(22.6 \%)$ patients. Performance of sorafenib appeared to lie between that of the aforementioned molecules. Again, caution should be exercised with this drug, due to the lack of controlled trials.

Interestingly, a follow-up analysis of the cabozantinib phase III study, showed a non-significant increase of 5.5 months in overall survival (26.6 in cabozantinib- vs. 21.1 in placebo-treated patients) [44]. It is, however, impor- tant to note that neither of the phase III trials were powered to measure overall survival. With MTC being a rare disease, accruing a trial to study overall survival is not feasible given the number of patients and time needed for statistical power. Still, in exploratory analyses, the overall survival was statistically longer in patients carrying the RET M918T mutation (44.3 months in cabozantinib vs. 18.9 months in placebo). This observation is important since future research should focus on identifying and validating prognostic biomarkers of response to treatment. Furthermore, a new important study suggests that younger age and recruitment for symptoms are indicators of an even more prolonged response, exceeding 48 months [45]. Nevertheless, the exact place of TKIs in the treatment of MTC remains unclear. The findings of this metaanalysis suggest that these drugs might be useful for controlling the disease by halting its progression. However, the positive effects should be counterbalanced with the significant toxicity in a shared patient-physician decision-making, particularly since to date no drug has a proven effect on the overall survival of these patients [46]. It is important to stress that all these studies of TKIs have provided us with abundant experience for better management of side effects aiming at improved quality of life; it is likely that this initial experience with the use of these drugs and their side effects will help us acknowledge their potential and improve the management of progressive MTC, by recognizing possible predictive factors of better response. In parallel, next-generation selective TKIs are being developed with the hope for an improved outcome and fewer AEs $[4,46]$.

Several limitations should be acknowledged in this study, as is the case with similar meta-analyses, in addition to the fact that for some of the TKI molecules the number of studies is very small. First, selected studies are characterized by significant heterogeneity. This might be attributed to differences in the definition of PD and differences in the time lapse between the diagnosis of DP and TKI initiation. Furthermore, some of finally selected studies had missing data, G3AEs and PFS included, mainly in observational, retrospective and phase II trials. We have chosen to include all studies in order to avoid a systematic selection bias error. Included studies had missing data, but this is the definition of real-life clinical data.

Second, there are differences in the follow-up length. The duration of follow-up was limited in most studies, not reaching a final point such as death; therefore, median overall survival could not be estimated.

Third, quality of life was not taken into account in some of the studies, though this would have been an im- 
portant observation from a clinical perspective. Since no difference in overall survival has been shown for any of the molecules studied so far, any improvement in quality of life would have been a strong argument for the use of these drugs in advanced MTC.

Fourth, RECIST criteria for DP may not have been correctly and independently evaluated in all studies as is the case in randomized phase III trials. Some of the eligible studies consist of small retrospective cohorts, but again ruling them out of the meta-analysis would have resulted in greater bias.

Finally, it should be stressed that head-to-head comparison between the different molecules has not been performed and thus, inevitably, the evidence is observational. Furthermore, the majority of studies were not placebo controlled, and the subset of them which were RCTs, were treated as observational since only the data from the treatment arm were included in the main analyses. This fact, along with the different design of the 2 phase III studies, makes it difficult to safely compare the different drug molecules regarding either efficacy or toxicity. However, for the 2 phase III RCTs, in the population of MTC patients treated with TKIs (either cabozantinib or vandetanib), a clinically pertinent benefit was observed (HR from 0.28 to 0.35 ) as compared to placebo.

In the present study, we have attempted to give a view of the current status of TKI use in MTC, as reflected in the published literature. A recent, strict meta-analysis inquiring the effect of TKIs on PFS, combined with a network meta-analysis, included only the 2 phase III RCT trials [47], and showed a comparable efficacy.

In conclusion, the use of TKIs in the management of advanced MTC has so far led to moderate therapeutic benefit, translating into either partial disease regression or stability in $73 \%$ of patients. However, the decision to initiate TKI treatment in MTC, should be made on an individual basis since the toxicity of these drugs is not negligible. Hopefully, the ongoing trials with novel molecules will prove even more effective.

\section{Acknowledgement}

The Greek School of Endocrinology initiative received support from Ipsen for the statistical analysis associated with the manuscript. The development at all stages of the concept and content of this paper was the sole responsibility of the authors.

\section{Statement of Ethics}

Ethical approval was not required as this is a systematic review/ meta-analysis.

\section{Conflict of Interest Statement}

S.T. has participated in educational, research or advisory activities supported by Amgen, Astra-Zeneca, Ipsen, Novartis, and Sanofi-Aventis. M.A. has served in the advisory board for the use of vandetanib in MTC and has received lecture fees from Sanofi. All other authors have no conflict of interest.

\section{Funding Sources}

There are no funding sources to declare.

\section{Author Contributions}

All authors participated in the Greek School of Endocrinology, an educational and research activity supported by Ipsen. Z.A.E., C.T., A.B., V.D., K.K., G.N., L.P. and S.T. have contributed to the selection of the studies included in this meta-analysis, the generation of data extracted from these studies, and have read and commented on drafts of this article. C.T. and K.T. did the statistical analysis, and C.T. completed the presentation of results. Z.A.E. and C.T. prepared the manuscript. M.A. and K.P.P. participated in the study design and reviewed the final manuscript. All authors have judged the quality of the evidence and strength of the data extracted from the studies, have contributed to the design and purpose of the meta-analysis, and have read and commented on the final draft of the manuscript.

\section{References}

1 Wells SA Jr, Asa SL, Dralle H, Elisei R, Evans DB, Gagel RF, et al. Revised American Thyroid Association guidelines for the management of medullary thyroid carcinoma. Thyroid. 2015 Jun;25(6):567-610.

2 Hadoux J, Pacini F, Tuttle RM, Schlumberger M. Management of advanced medullary thyroid cancer. Lancet Diabetes Endocrinol. 2016 Jan;4(1):64-71.

TKIs in Medullary Thyroid Cancer
3 Spitzweg C, Morris JC, Bible KC. New drugs for medullary thyroid cancer: new promises? Endocr Relat Cancer. 2016 Jun;23(6):R28797.

4 Ilanchezhian M, Khan S, Okafor C, Glod J, Del Rivero J. Update on the Treatment of Medullary Thyroid Carcinoma in Patients with Multiple Endocrine Neoplasia Type 2. Horm Metab Res. 2020, Online ahead of print.
5 Elisei R, Romei C, Cosci B, Agate L, Bottici V, Molinaro E, et al. RET genetic screening in patients with medullary thyroid cancer and their relatives: experience with 807 individuals at one center. J Clin Endocrinol Metab. 2007 Dec;92(12):4725-9.

6 Ye L, Santarpia L, Gagel RF. The evolving field of tyrosine kinase inhibitors in the treatment of endocrine tumors. Endocr Rev. 2010 Aug; 31(4):578-99. 
7 Bible KC, Ryder M. Evolving molecularly targeted therapies for advanced-stage thyroid cancers. Nat Rev Clin Oncol. 2016 Jul;13(7): 403-16.

8 Klein Hesselink EN, Steenvoorden D, Kapiteijn E, Corssmit EP, van der Horst-Schrivers AN, Lefrandt JD, et al. Therapy of endocrine disease: response and toxicity of small-molecule tyrosine kinase inhibitors in patients with thyroid carcinoma: a systematic review and meta-analysis. Eur J Endocrinol. 2015 May;172(5):R215-25.

9 Elisei R, Schlumberger MJ, Müller SP, Schöffski P, Brose MS, Shah MH, et al. Cabozantinib in progressive medullary thyroid cancer. J Clin Oncol. 2013 Oct;31(29):3639-46.

10 Eisenhauer EA, Therasse P, Bogaerts J, Schwartz LH, Sargent D, Ford R, et al. New response evaluation criteria in solid tumours: revised RECIST guideline (version 1.1). Eur J Cancer. 2009 Jan;45(2):228-47.

11 ClinicalTrials.gov. [Internet]. Bethesda (MD): National Library of Medicine (US). Identifier NCT01496313, To Compare The Effects Of Two Doses Of Vandetanib In Patients With Advanced Medullary Thyroid Cancer [Online]; November 2017, [cited 2018 May]; Available from: https://ClinicalTrials. gov/show/NCT01496313.

12 Gross DJ, Munter G, Bitan M, Siegal T, Gabizon A, Weitzen R, et al.; Israel Glivec in Solid Tumors Study Group. The role of imatinib mesylate (Glivec) for treatment of patients with malignant endocrine tumors positive for c-kit or PDGF-R. Endocr Relat Cancer. 2006 Jun;13(2):535-40.

13 de Groot JW, Zonnenberg BA, van UffordMannesse PQ, de Vries MM, Links TP, Lips CJ, et al. A phase II trial of imatinib therapy for metastatic medullary thyroid carcinoma. J Clin Endocrinol Metab. 2007 Sep;92(9): 3466-9.

14 Frank-Raue K, Fabel M, Delorme S, Haberkorn U, Raue F. Efficacy of imatinib mesylate in advanced medullary thyroid carcinoma. Eur J Endocrinol. 2007 Aug;157(2):215-20.

15 Cohen EE, Rosen LS, Vokes EE, Kies MS, Forastiere AA, Worden FP, et al. Axitinib is an active treatment for all histologic subtypes of advanced thyroid cancer: results from a phase II study. J Clin Oncol. 2008 Oct;26(29):470813.

16 Schlumberger MJ, Elisei R, Bastholt L, Wirth LJ, Martins RG, Locati LD, et al. Phase II study of safety and efficacy of motesanib in patients with progressive or symptomatic, advanced or metastatic medullary thyroid cancer. J Clin Oncol. 2009 Aug;27(23):3794-801.

17 Carr LL, Mankoff DA, Goulart BH, Eaton KD, Capell PT, Kell EM, et al. Phase II study of daily sunitinib in FDG-PET-positive, iodinerefractory differentiated thyroid cancer and metastatic medullary carcinoma of the thyroid with functional imaging correlation. Clin Cancer Res. 2010 Nov; 16(21):5260-8.
18 Lam ET, Ringel MD, Kloos RT, Prior TW, Knopp MV, Liang J, et al. Phase II clinical trial of sorafenib in metastatic medullary thyroid cancer. J Clin Oncol. 2010 May;28(14): 2323-30.

19 Robinson BG, Paz-Ares L, Krebs A, Vasselli J, Haddad R. Vandetanib $(100 \mathrm{mg})$ in patients with locally advanced or metastatic hereditary medullary thyroid cancer. J Clin Endocrinol Metab. 2010 Jun;95(6):2664-71.

20 Wells SA Jr, Gosnell JE, Gagel RF, Moley J, Pfister D, Sosa JA, et al. Vandetanib for the treatment of patients with locally advanced or metastatic hereditary medullary thyroid cancer. J Clin Oncol. 2010 Feb;28(5):767-72.

21 Ahmed M, Barbachano Y, Riddell A, Hickey J, Newbold KL, Viros A, et al. Analysis of the efficacy and toxicity of sorafenib in thyroid cancer: a phase II study in a UK based population. Eur J Endocrinol. 2011 Aug; 165(2):31522.

22 Frank-Raue K, Ganten M, Kreissl MC, Raue F. Rapid response to sorafenib in metastatic medullary thyroid carcinoma. Exp Clin Endocrinol Diabetes. 2011 Mar;119(3):151-5.

23 Hong DS, Cabanillas ME, Wheler J, Naing A, Tsimberidou AM, Ye L, et al. Inhibition of the Ras/Raf/MEK/ERK and RET kinase pathways with the combination of the multikinase inhibitor sorafenib and the farnesyltransferase inhibitor tipifarnib in medullary and differentiated thyroid malignancies. J Clin Endocrinol Metab. 2011 Apr;96(4):997-1005.

24 Kurzrock R, Sherman SI, Ball DW, Forastiere AA, Cohen RB, Mehra R, et al. Activity of XL184 (Cabozantinib), an oral tyrosine kinase inhibitor, in patients with medullary thyroid cancer. J Clin Oncol. 2011 Jul;29(19): 2660-6.

25 Capdevila J, Iglesias L, Halperin I, Segura A, Martínez-Trufero J, Vaz MA, et al. Sorafenib in metastatic thyroid cancer. Endocr Relat Cancer. 2012 Apr;19(2):209-16.

26 Wells SA Jr, Robinson BG, Gagel RF, Dralle $\mathrm{H}$, Fagin JA, Santoro M, et al. Vandetanib in patients with locally advanced or metastatic medullary thyroid cancer: a randomized, double-blind phase III trial. J Clin Oncol. 2012 Jan;30(2):134-41.

27 Bible KC, Suman VJ, Molina JR, Smallridge RC, Maples WJ, Menefee ME, et al.; Endocrine Malignancies Disease Oriented Group, Mayo Clinic Cancer Center, and the Mayo Phase 2 Consortium. A multicenter phase 2 trial of pazopanib in metastatic and progressive medullary thyroid carcinoma: $\mathrm{MC} 057 \mathrm{H}$. J Clin Endocrinol Metab. 2014 May;99(5): 1687-93.

28 Locati LD, Licitra L, Agate L, Ou SH, Boucher A, Jarzab B, et al. Treatment of advanced thyroid cancer with axitinib: Phase 2 study with pharmacokinetic/pharmacodynamic and quality-of-life assessments. Cancer. 2014 Sep; 120(17):2694-703
29 Massicotte MH, Brassard M, Claude-Desroches $\mathrm{M}$, Borget I, Bonichon F, Giraudet AL, et al. Tyrosine kinase inhibitor treatments in patients with metastatic thyroid carcinomas: a retrospective study of the TUTHYREF network. Eur J Endocrinol. 2014 Mar;170(4): 575-82.

30 Benekli M, Yalcin S, Ozkan M, Elkiran ET, Sevinc A, Cabuk D, et al. Efficacy of sorafenib in advanced differentiated and medullary thyroid cancer: experience in a Turkish population. OncoTargets Ther. 2014 Dec;8:1-5.

31 Chougnet CN, Borget I, Leboulleux S, de la Fouchardiere C, Bonichon F, Criniere L, et al. Vandetanib for the treatment of advanced medullary thyroid cancer outside a clinical trial: results from a French cohort. Thyroid. 2015 Apr;25(4):386-91.

32 Chrisoulidou A, Mandanas S, Margaritidou E, Mathiopoulou L, Boudina M, Georgopoulos $\mathrm{K}$, et al. Treatment compliance and severe adverse events limit the use of tyrosine kinase inhibitors in refractory thyroid cancer. Onco Targets Ther. 2015 Sep;8:2435-42.

33 Lim SM, Chung WY, Nam KH, Kang SW, Lim JY, Kim HG, et al. An open label, multicenter, phase II study of dovitinib in advanced thyroid cancer. Eur J Cancer. 2015 Aug; 51(12):1588-95.

34 Werner RA, Schmid JS, Muegge DO, Lückerath K, Higuchi T, Hänscheid H, et al. Prognostic Value of Serum Tumor Markers in Medullary Thyroid Cancer Patients Undergoing Vandetanib Treatment. Medicine (Baltimore). 2015 Nov;94(45):e2016

35 de Castroneves LA, Negrão MV, de Freitas RM, Papadia C, Lima JV Jr, Fukushima JT, et al. Sorafenib for the Treatment of Progressive Metastatic Medullary Thyroid Cancer: Efficacy and Safety Analysis. Thyroid. 2016 Mar; 26(3):414-9.

36 Schlumberger M, Jarzab B, Cabanillas ME, Robinson B, Pacini F, Ball DW, et al. A Phase II Trial of the Multitargeted Tyrosine Kinase Inhibitor Lenvatinib (E7080) in Advanced Medullary Thyroid Cancer. Clin Cancer Res. 2016 Jan;22(1):44-53.

37 Tiedje V, Ting S, Walter RF, Herold T, Worm $\mathrm{K}$, Badziong J, et al. Prognostic markers and response to vandetanib therapy in sporadic medullary thyroid cancer patients. Eur J Endocrinol. 2016 Sep;175(3):173-80.

38 Capdevila J, Trigo JM, Aller J, Manzano JL, Adrián SG, Llopis CZ, et al. Axitinib treatment in advanced RAI-resistant differentiated thyroid cancer (DTC) and refractory medullary thyroid cancer (MTC). Eur J Endocrinol. 2017 Oct;177(4):309-17.

39 Ito Y, Onoda N, Ito KI, Sugitani I, Takahashi $\mathrm{S}$, Yamaguchi I, et al. Sorafenib in Japanese Patients with Locally Advanced or Metastatic Medullary Thyroid Carcinoma and Anaplastic Thyroid Carcinoma. Thyroid. 2017 Sep; 27(9):1142-48 
40 Ravaud A, de la Fouchardiere C, Caron P, Doussau A, Do Cao C, Asselineau J, et al. A multicenter phase II study of sunitinib in patients with locally advanced or metastatic differentiated, anaplastic or medullary thyroid carcinomas: mature data from the THYSU study. Eur J Cancer. 2017 May;76:110-7.

41 Uchino K, Komoda M, Tomomatsu J, Okamoto T, Horiuchi K, Tsuji A, et al. Safety and tolerability of vandetanib in Japanese patients with medullary thyroid cancer: a phase I/II open-label study. Endocr Pract. 2017 Feb; 23(2):149-56.

42 Kraft IL, Akshintala S, Zhu Y, Lei H, DerseAnthony C, Dombi E, et al. Outcomes of Children and Adolescents with Advanced Hereditary Medullary Thyroid Carcinoma Treated with Vandetanib. Clin Cancer Res. 2018 Feb; 24(4):753-65.
43 Trimboli P, Castellana M, Virili C, Giorgino F, Giovanella L. Efficacy of Vandetanib in Treating Locally Advanced or Metastatic Medullary Thyroid Carcinoma According to RECIST Criteria: A Systematic Review and Meta-Analysis. Front Endocrinol (Lausanne). 2018 May;9:224.

44 Schlumberger M, Elisei R, Müller S, Schöffski $\mathrm{P}$, Brose M, Shah M, et al. Overall survival analysis of EXAM, a phase III trial of cabozantinib in patients with radiographically progressive medullary thyroid carcinoma. Ann Oncol. 2017 Nov;28(11):2813-9.
45 Valerio L, Bottici V, Matrone A, Piaggi P, Viola D, Cappagli V, et al. Medullary thyroid cancer treated with vandetanib: predictors of a longer and durable response. Endocr Relat Cancer. 2020 Jan;27(2):97-110.

46 Ceolin L, Duval MA, Benini AF, Ferreira CV Maia AL. Medullary thyroid carcinoma beyond surgery: advances, challenges, and perspectives. Endocr Relat Cancer. 2019 Aug; 26(9):R499-518.

47 Tappenden P, Carroll C, Hamilton J, Kaltenthaler E, Wong R, Wadsley J, et al. Cabozantinib and vandetanib for unresectable locally advanced or metastatic medullary thyroid cancer: a systematic review and economic model. Health Technol Assess. 2019 Feb; 23(8):1-144. 